\title{
Near Infrared Plasmonic Gas Sensing with Doped Metal Oxide Nanocrystals ${ }^{\dagger}$
}

\author{
Marco Sturaro ${ }^{1}$, Enrico Della Gaspera ${ }^{2}$, Carlo Cantalini ${ }^{3}$, Massimo Guglielmi ${ }^{1}$ \\ and Alessandro Martucci ${ }^{1, *}$ \\ 1 Dipartimento di Ingegneria Industriale, Università di Padova, Padova, Italy; sturarom@gmail.com (M.S.); \\ massimo.guglielmi@unipd.it (M.G.) \\ 2 School of Science, RMIT University, Melbourne, VIC, Australia; enrico.dellagaspera@rmit.edu.au \\ 3 Dipartimento di Ingegneria Industriale, Università di L'Aquila, L'Aquila, Italy; carlo.cantalini@univaq.it \\ * Correspondence: alex.martucci@unipd.it \\ † Presented at the Eurosensors 2017 Conference, Paris, France, 3-6 September 2017.
}

Published: 5 September 2017

\begin{abstract}
In this paper, we demonstrate the application of $\mathrm{ZnO}$ doped with gallium (GZO), aluminum (AZO) and germanium (GeZO) nanocrystals as novel plasmonic and chemiresistive sensors for the detection of hazardous gases including hydrogen $\left(\mathrm{H}_{2}\right)$ and nitrogen dioxide $\left(\mathrm{NO}_{2}\right)$. GZO, AZO and GeZO nanocrystals are obtained by non-aqueous colloidal heat-up synthesis with high transparency in the visible range and strong localized surface plasmon resonance (LSPR) in the near IR range, tunable with dopant concentration (up to $20 \% \mathrm{~mol}$ nominal). Thanks to the strong sensitivity of the LSPR to chemical and electrical changes occurring at the surface of the nanocrystals, such optical features can be used to detect the presence of toxic gases. By monitoring the changes in the dopant-induced plasmon resonance in the near infrared, we demonstrate that GZO, AZO and GeZO thin films prepared depositing an assembly of highly doped ZnO colloids are able to optically detect both oxidizing and reducing gases at mild $\left(<100{ }^{\circ} \mathrm{C}\right)$ operating temperatures. Combined optical and electrical measurements show that the dopants within $\mathrm{ZnO}$ nanocrystals enhance the gas sensing response compared to undoped $\mathrm{ZnO}$.
\end{abstract}

Keywords: transparent conductive oxides; doped zinc oxide; optical gas sensors

\section{Introduction}

$\mathrm{ZnO}$ is one of the most studied materials for gas sensing applications because of its chemical sensitivity to many analytes and high thermal. Doping has been shown to influence the optical and electrical properties of $\mathrm{ZnO}$, with beneficial effects for its catalytic and sensing performances [1]. In particular, trivalent cations (i.e., $\mathrm{Al}^{3+}$ and $\mathrm{Ga}^{3+}$ ) as substitutional dopants for zinc have been extensively studied, mostly for application in transparent conductors (TCO). These dopants are also responsible for the generation of a strong localized surface plasmon resonance (LSPR) in the near infrared, arising from the increased free electron concentration. While the main applications of TCOs are in optoelectronic devices, they have been recently used also for gas sensing applications, in particular $\mathrm{ZnO}$ doped with gallium (GZO) and aluminum (AZO) have been used for electrical sensing of hydrogen, methanol, nitrogen dioxide, hydrogen sulfide and optical sensing of hydrogen [2]. However, the optical features provided by the LSPR have never been used as sensing platforms.

In this paper, we demonstrate plasmonic gas sensing using the IR LSPR peak of GZO, AZO and $\mathrm{ZnO}$ doped with Germanium (GeZO) films. Aliovalent dopants in $\mathrm{ZnO}$ induce the formation of a IR LSPR peak, which analogously to visible LSPR peak of metal nanoparticles, is affected by the presence of both reducing and oxidizing gases. We elucidated the role of the different dopants, oxygen vacancies and overall sensing mechanism combining the optical gas sensing data with electrical 
measurements. Moreover, by exposing the doped $\mathrm{ZnO}$ film to blue light during the sensing tests, we achieved room temperature sensitivity to sub-ppm $\mathrm{NO}_{2}$ concentrations.

\section{Materials and Methods}

Zinc oxide nanocrystals (NCs) were synthetized through a previously reported heat-up colloidal method [3]. In a typical synthesis of undoped $\mathrm{ZnO}$ NCs, $1.26 \mathrm{~g} \mathrm{Zn}$ stearate $\left(\mathrm{ZnSt}_{2}\right), 3.2 \mathrm{~g}$ 1-dodecanol (1-DDOL) and $1.76 \mathrm{~g}$ oleic acid (OA) were mixed togheter in a round bottom flask, using $21 \mathrm{~mL}$ 1-Octadecene (ODE) as non-coordinating solvent. To obtain doped nanoparticles, a certain amount of Gallium acetylacetonate, Aluminum acetylacetonate or Germanium alkoxides was added. The solution was first heated to $130{ }^{\circ} \mathrm{C}$, then it was maintained at this temperature for $45 \mathrm{~min}$, and eventually it was heated at $10{ }^{\circ} \mathrm{C} / \mathrm{min}$ to $240{ }^{\circ} \mathrm{C}$ under strong stirring. After $3 \mathrm{~h}$ at $240{ }^{\circ} \mathrm{C}$ to ensure efficient dopant incorporation, the solution was cooled down to room temperature under nitrogen. The NCs were then collected and purified. After purification, the NCs were redispersed in octane or toluene at a concentration of $\sim 100 \mathrm{mg} / \mathrm{mL}$. Spin coated films were deposited from these concentrated solutions at $1000 \mathrm{rpm}$ for $30 \mathrm{~s}$, and then stabilized on an hot plate at $150{ }^{\circ} \mathrm{C}$ for $15 \mathrm{~min}$ in air. Thin films were then treated following a procedure previously described by our group in a $5 \% \mathrm{H}_{2}-95 \% \mathrm{Ar}$ gas mixture.

X-ray diffraction (XRD) analyses on dry nanocrystalline powders were performed using a Bruker D8 Advance diffractometer using CuK $(0.15418 \mathrm{~nm})$ radiation at $40 \mathrm{kV}$ and $40 \mathrm{~mA}$; XRD analyses on thin films were performed using a Philips PW1710 diffractometer equipped with glancing-incidence X-ray optics. Optical absorption spectra of samples were obtained in the 200-2500 nm range using a Jasco V-570 standard spectrophotometer. Optical gas sensing tests were performed by making optical absorption measurements in the 350-2500 nm wavelength range on films deposited on $\mathrm{SiO}_{2}$ substrates using a Harrick gas flow cell. The standard operating temperature (OT) was set between 80 and $200{ }^{\circ} \mathrm{C}$ and gases at concentrations of $1 \mathrm{vol} \%$ for $\mathrm{H}_{2}$ and of $1000 \mathrm{ppm}$ for $\mathrm{NO}_{2}$ in dry air at a flow rate of $0.4 \mathrm{~L} / \mathrm{min}$ were used. The incident spectrophotometer beam was set normal to the film surface and illuminated an area of $\sim 13 \mathrm{~mm}^{2}$.

Electrical gas sensing tests were obtained on films deposited onto a $\mathrm{Si}_{1} \mathrm{Si}_{3} \mathrm{~N}_{4}$ supports, inserted in a chamber provided with Teflon tubing and atmospheric discharge. Gases were mixed in a MKS147 multigas mass controller to obtain final gas target concentrations in the ranges $20 \mathrm{ppb}-1 \mathrm{ppm}$ of $\mathrm{NO}_{2}$ and $50-1000 \mathrm{ppm}$ of $\mathrm{H}_{2} . \mathrm{NO}_{2}$ gas concentrations in the downstream were measured by an Ansyco AS32M chemiluminescence analyser from Environnement S.A.

\section{Results and Discussion}

ZnO NCs with different doping levels were synthesized by changing the amount of the dopants precursor in the reaction mixture. As demonstrated previously [4], the presence of the aliovalent dopant triggers the formation of a surface plasmon resonance in the near infrared, as evident from Figure 1a. The IR signatures of GZO NCs are strongly dependent on the Ga doping, which also slightly affects the absorption properties in the visible spectrum, giving the doped NCs a distinctive green-blue color, with respect to the uncolored pure $\mathrm{ZnO}$ (inset of Figure 1a). The GZO NCs synthesized here are all crystalline in hexagonal wurtzite structure (ICDD No. 36-1451) without any impurities or particular preferred orientation, as shown in Figure 1b. Consistent with previous data [4], due to the similar ionic radii of zinc and gallium, no shift of the diffraction peaks between doped and undoped $\mathrm{ZnO}$ can be detected. The crystallite diameter of the NCs evaluated from the diffraction patterns using the Scherrer equation is $\sim 10 \mathrm{~nm}$, and becomes slightly larger with increased dopant concentration. This is consistent with the previously observed role of dopant ions in defining shape and dimension in colloidal NCs. 

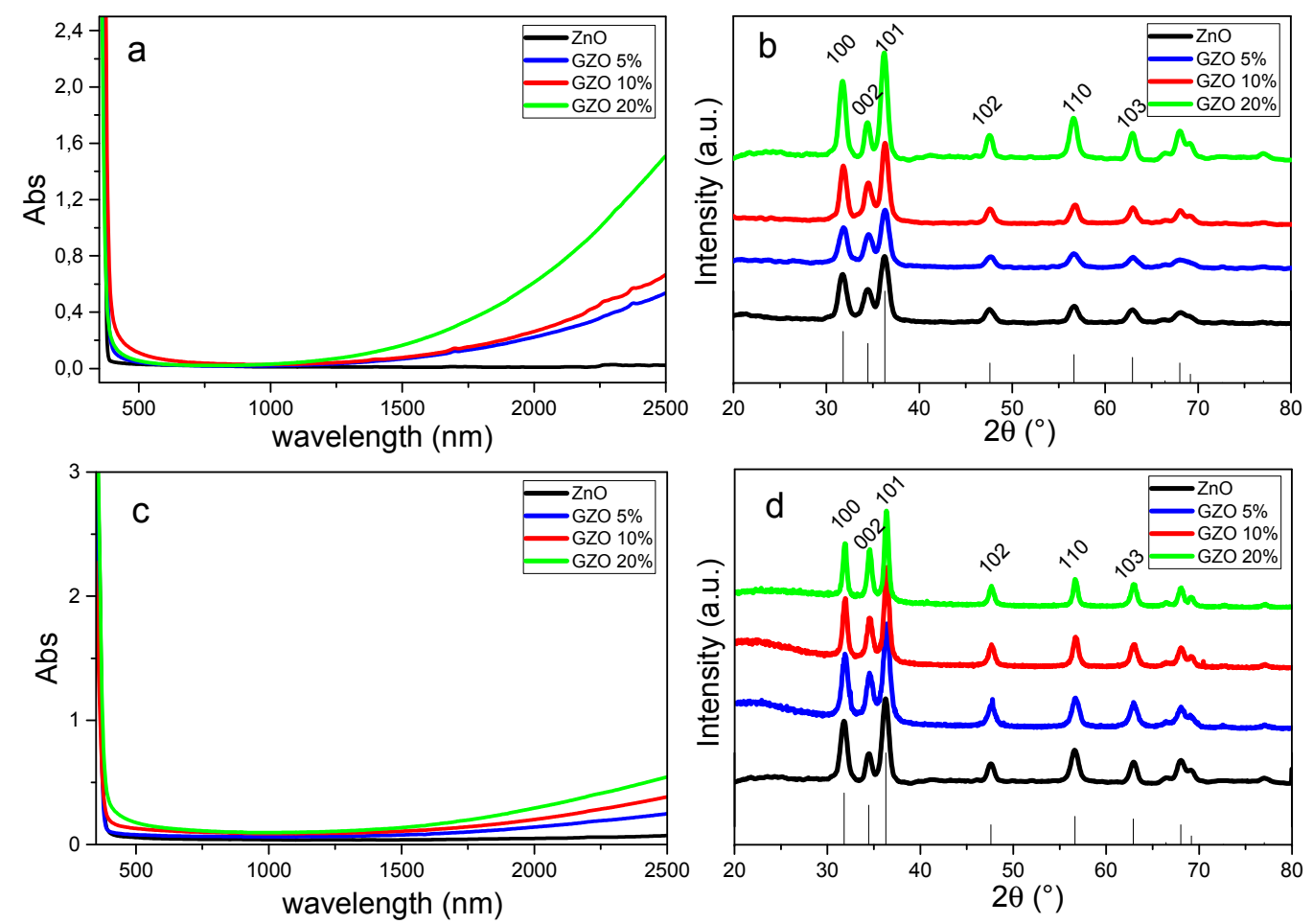

Figure 1. Optical absorption spectra $(\mathbf{a}, \mathbf{c})$ and XRD patterns $(\mathbf{b}, \mathbf{d})$ for the as-synthesized GZO $\mathrm{NCs}(\mathbf{a}, \mathbf{b})$ and for thin films after annealing at $450{ }^{\circ} \mathrm{C}$ in forming gas $(\mathbf{c}, \mathbf{d})$ at different nominal gallium doping. The absorption spectra of the NCs in solution are obtained from equimolar colloidal suspensions using TCE as solvent.

Despite these small differences in size, all the synthesized NCs can be suspended in non-polar solvents at high concentration obtaining highly stable colloidal "inks" that are suitable for thin films deposition. The films were prepared via spin coating as described in the Experimental section and subsequently annealed in reducing atmosphere at $450{ }^{\circ} \mathrm{C}$. Prior to such annealing, the samples were exposed to UV light for $30 \mathrm{~min}$, to remove organic compounds-mainly composed of surface ligands-and obtain purely inorganic coatings, as previously demonstrated. After such annealing procedure, GZO films that are transparent in the visible range and show a marked infrared absorption were obtained. Consistent with what was observed for the as-synthesized NCs, the NIR absorption progressively increases with increased Ga doping (Figure 1c). Similar results have been obtained also for the AZO and GeZO film.

We analyzed the variation in the LSPR peak for GZO films exposed to $1 \%$ vol $\mathrm{H}_{2}$ and $1000 \mathrm{ppm}$ $\mathrm{NO}_{2}$ at different operating temperatures in the range $25-150{ }^{\circ} \mathrm{C}$ and dry air as carrier gas. Higher OT ( $\mathrm{T}>200^{\circ} \mathrm{C}$ ) were found to drastically decrease the plasmonic absorption due to decrease in oxygen vacancies. After exposure to hydrogen (a reducing gas), the absorbance in the NIR range increases, while upon exposure to $1000 \mathrm{ppm} \mathrm{NO}$ (an oxidizing gas) a decrease in optical absorption in the NIR is observed. To highlight this difference, Figure 2 also shows the Optical Absorbance Change parameter, which is defined as the difference between absorbance during gas exposure and absorbance in air $(\mathrm{OAC}=\mathrm{Absgas}-\mathrm{Absair})$.

These behaviours are consistent with IR plasmonic resonance shifts due to electron density variations and band gap shifts in $n$-type semiconductor like $\mathrm{ZnO}$, caused by Bursnstein-Moss effect. In the case of hydrogen, electrons are injected in the metal oxide according to reaction (2) causing an increase in the concentration of free carriers which genereates a consequent blue shift of the LSPR peak and an increase of the optical band gap. The interaction with oxidizing gases like $\mathrm{NO}_{2}$ conversely causes the removal of electrons acording to reaction (3), with the related red-shift of the plasmon peak and the reduction of the optical band gap. These related LSPR shifts, driven by different oxidising/reduging gases, represents a great opportunity to improve sensor selectivity. Test 
could be performed exploiting the variation in absorbance both in N-IR range than band gap region. However, the possibility to obtain results due to N-IR LSPR shifts represents an important goal, because it permits to explore the behavior of absorptive gases in visible range (like $\mathrm{NO}_{2}$ ) maintaining sensor's high transparency.

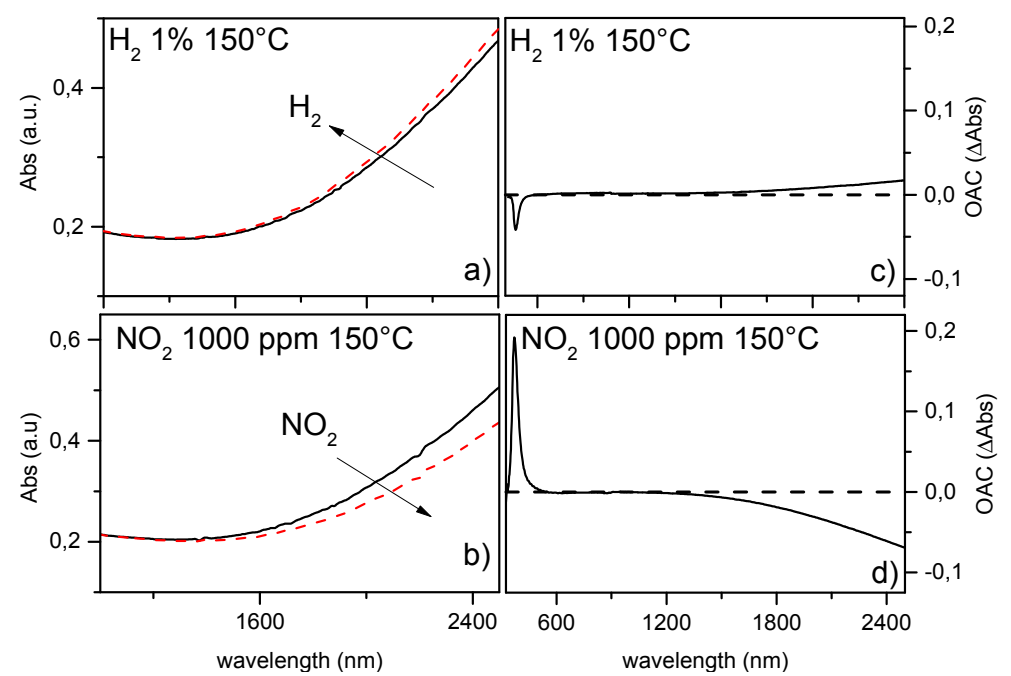

Figure 2. Optical absorption spectra for GZO10 sample when exposed to air (black solid lines) and (a) $\mathrm{H}_{2}$ 1\% vol or (b) $\mathrm{NO}_{2} 1000$ ppm (red dashed lines) at $150{ }^{\circ} \mathrm{C}$ OT. (c,d) show optical absorbance change $(\mathrm{OAC}=\mathrm{Absgas}-\mathrm{Absair})$ for $\mathrm{GZO} 10$ at $150{ }^{\circ} \mathrm{C}$ when exposed to $\mathrm{H}_{2}$ and $\mathrm{NO}_{2}$, respectively.

The chemoresistive response of the GZO; $\mathrm{AZO}$ and $\mathrm{GeZO}$ films to $\mathrm{NO}_{2}$ and $\mathrm{H}_{2}$ gases was also investigated and compared with optical gas sensing measurements. We explored the effect of both temperature (OT range $25-100{ }^{\circ} \mathrm{C}$ ), and visible light irradiation using purple-blue (PB) LED source $\left(\lambda=430 \mathrm{~nm}\right.$ wavelength and $770 \mu \mathrm{W} / \mathrm{cm}^{2}$ intensity). The resistance change during hydrogen exposure was always lower with respect to $\mathrm{NO}_{2}$ exposure, for all the conditions. This behavior is in line with the smaller gas sensing response to $\mathrm{H}_{2}$ as respect to $\mathrm{NO}_{2}$ observed during optical sensing tests.

Irradiation with $\mathrm{PB}$ light enhances both response and recovery time, as respect to dark conditions. Given the positive effect of PB light irradiation, reasonable gas sensing features are maintained even at $50{ }^{\circ} \mathrm{C} \mathrm{OT}$, yielding for the $20 \%$ GZO very good sensing performances even at low (250 ppb) $\mathrm{NO}_{2}$ concentration.

Conflicts of Interest: The authors declare no conflict of interest.

\section{References}

1. Etacheri, V.; Roshan R.; Kumar, V. Mg-Doped ZnO Nanoparticles for Efficient Sunlight-Driven Photocatalysis. ACS Appl. Mater. Interfaces 2012, 4, 2717-2725.

2. Hou, Y.; Soleimanpour, A.M.; Jayatissa, A.H. Low Resistive Aluminum Doped Nanocrystalline Zinc Oxide for Reducing Gas Sensor Application via Sol-Gel Process. Sens. Actuators B 2013, 177, 761-769.

3. Sturaro, M.; Della Gaspera, E.; Michieli, N.; Cantalini, C.; Emamjomeh, S.M.; Guglielmi, M.; Martucci, A. Degenerately Doped Metal Oxide Nanocrystals as Plasmonic and Chemoresistive Gas Sensors. ACS Appl. Mater. Interfaces 2016, 8, 30440-30448.

4. Gaspera, E.; Bersani, M.; Cittadini, M.; Guglielmi, M.; Pagani, D.; Noriega, R.; Mehrall, S.; Salleoll, A.; Martucci, A. Low-temperature processed Ga-doped ZnO coatings from colloidal inks. J. Am. Chem. Soc. 2013, 135, 3439-3448.

(C) 2017 by the authors. Licensee MDPI, Basel, Switzerland. This article is an open access article distributed under the terms and conditions of the Creative Commons Attribution (CC BY) license (http://creativecommons.org/licenses/by/4.0/). 